\title{
Migraciones y agua en la Frontera entre MéXiCO, GUATEMALA Y BELICE: APROXIMACIONES EN TORNO A UNA RELACIÓN MULTIFORME
}

\author{
Edith F. Kauffer Michel
}

Resumen: La frontera sur de México constituye un escenario de complejos flujos de migración entre los cuales encontramos movimientos internos e internacionales que obedecen a diversos factores. Asimismo, en la región, el recurso hídrico se caracteriza por su abundancia debido a fuertes precipitaciones concentradas en pocos meses del año que provocan una presencia multiforme de fuentes de agua superficiales y subterráneas. Este trabajo propone explorar de manera preliminar las relaciones entre estas dos realidades que se entrecruzan en un mismo territorio. A partir de varias definiciones del concepto de frontera, analiza en un primer momento la relación entre las aguas y las dos principales dimensiones de la frontera en la región estudiada. Posteriormente, evoca la presencia de agua como un elemento favorable y desfavorable para las migraciones, cuya existencia ejerce atracción, pero cuya abundancia origina desplazamientos y finalmente cuya presencia en la frontera sur facilita los flujos migratorios pero acentúa los riesgos enfrentados por los migrantes.

Palabras clave: Migraciones, agua, fronteras, México, Guatemala, Belice.

Enviado a dictamen: 18 de junio de 2010

Aprobación: 06 de septiembre de 2010

Revisiones: 1

Edith F. Kauffer Michel, Doctora en Ciencias Políticas por el Instituto de Estudios Políticos de las Universidad de Aix-Marseille III, Francia. Actualmente labora en Centro de Investigaciones y Estudios Superiores en Antropología Social (CIESAS)-Sureste. San Cristóbal de Las Casas, Chiapas. Temas de especialización: agua, políticas públicas, hidropolítica, frontera, relaciones internacionales, migraciones y género. Correo electrónico: ekauffer@ciesas.edu. mx; kauffer69@hotmail.com.
Abstract: The southern border of Mexico is a complex scenario of migration flows among them are internal and international movements are due to several factors. Also in the region, water resources are characterized by their abundance due to heavy rainfall concentrated in a few months of the year that cause a multifaceted presence of water sources and groundwater. This paper aims to explore in a preliminary way the relationship between these two realities intertwining in the same territory. Based on various definitions of the border, looks at first the relationship between water and the two main dimensions of the border in the region studied. Later, he evokes the presence of water as a favorable and unfavorable to migration, the existence of attraction exercises, but whose abundance shifts and eventually causes whose presence on the southern border facilitates migration but emphasizes the risks faced by migrants.

Keywords: Migrations, water, border, frontier, Mexico, Guatemala, Belize.

\section{Introducción}

$\mathrm{D}$ espués de más de dos décadas de estudios sistemáticos en torno a diversos fenómenos de la realidad de la frontera que México comparte con Guatemala y Belice, dicho espacio territorial ha cobrado cierta notoriedad dentro del ámbito académico en comparación con el olvido característico de décadas 
Migraciones y AGUA EN LA FRONTERA ENTRE MÉXICO,

anteriores. Sin embargo, cabe subrayar que la literatura actual evidencia cuatro grandes limitantes. La primera de ella es el predominio de una visión mexicana en torno a este espacio que se refleja no solamente en la denominación de "frontera sur", que remite al sur visto desde el centro de la República mexicana y por lo tanto excluye de facto la visión de los otros países, sino también en la escasez de trabajos producidos desde el sur - Guatemala y Belice - para los cuales la frontera común corresponde a su "frontera norte". La visión mexicana de dicha "frontera sur" predomina en torno a este espacio en tanto se trata de un Estado favorecido por una serie de asimetrías políticas, económicas y territoriales con respecto a sus vecinos, la cual se traduce en una hegemonía académica.

En este contexto, la denominación de frontera sur es asumida y retomada por casi todos los estudiosos de la región sin ser cuestionada por su visión excluyente (Ángeles et al., 2005; Basail, 2005; Hernández, 2005; Villafuerte, 2004) aun cuando los autores reconocen la necesidad y la pertinencia de tomar en cuenta la visión del otro lado (Castillo et al., 2006; De Vos, 2002a; Kauffer, 2002b; Morales, 2005) en una perspectiva más transfronteriza que fronteriza. Las excepciones a esta visión hegemónica son pocas (Dardón, 2002; Kauffer, 2005b; Valdez, 2006; Vautrevers, 2005). La segunda limitante de la literatura actual se caracteriza por una visión de la "frontera sur" centrada casi exclusivamente en la relación entre el estado de Chiapas y Guatemala y en menor medida entre Quintana Roo y Belice. Ello se debe principalmente a las recientes e inacabadas dinámicas fronterizas de los estados de Campeche —aún muy incipiente- - y de Tabasco - ya marcadas desde hace algunos años en cuanto a los flujos migratorios y a las relaciones comerciales-que Castillo et al. (2006: 21) atribuyen al carácter poco poblado de la parte guatemalteca que no favorece el desarrollo de relaciones transfronterizas. Hoy por hoy en la academia la visión de esta frontera sigue sin extenderse a la totalidad de su extensión geográfica.
La tercera limitante está relacionada con el hecho de que si bien los fenómenos sociales, políticos, económicos en torno a la frontera, que se han convertido en objeto de estudio, se han ampliado; entre estos sobresalen los estudios migratorios, las relaciones comerciales (Villafuerte, 2004), la conformación histórica (De Vos, 1993; Fábregas, 2005), y las dinámicas culturales que permiten el establecimiento de lazos de ambas partes (Cruz, 1998), otras realidades quedan ampliamente ignoradas aun por trabajos recientes que pretenden presentar una visión amplia de la frontera (Hernández, 2005; Castillo et al., 2006). Tal es el caso de los recursos hídricos en una región de abundancia natural que son abordados de forma muy marginal en gran parte de esta literatura o totalmente ignorados por ella.

La cuarta limitante es fundamental porque se centra en la indefinición de lo que los diferentes autores entienden como frontera; es decir, si esta corresponde a una delimitación político administrativa circunscrita a estados, departamentos, distritos o municipios que colindan con otro país, si se trata de una franja establecida arbitrariamente de ambos lados de la línea de división internacional, si remite a una región o a varias regiones (Castillo et al., 2006: 17-21, Fábregas, 2005 40-43), si refiere a delimitaciones geográficas — como las cuencas - o a otros tipos de procesos transfronterizos de corte social en constante elaboración (Castillo et al., 2006: 15; Limón, 2008). En este aspecto, las definiciones político jurídica, geográfica y más sociocultural de la frontera llevan a diferentes formas de conceptualizarla.

Las migraciones y la presencia de agua en la frontera que México comparte con Guatemala y Belice constituyen dos realidades que aparecen a primera vista desvinculadas. Ambas no son recientes ni novedosas, pero han adquirido últimamente dimensiones fundamentales para la región. No solamente el agua, en tanto recurso natural y como fuente de vida, medio de transporte y soporte de actividades humanas, y los protagonistas de la migración coinciden en el espacio fronterizo y transfronterizo, cruzan también la frontera 
política de forma paralela o conjunta y permiten entonces observar una serie de interacciones de diversa indole.

Este trabajo pretende relacionar un proceso bastante estudiado en la región, las migraciones, y otro poco abordado, el agua, en este espacio de múltiples coincidencias.

A partir de casi dos décadas centradas en la realización de proyectos de investigación en temas migratorios, 1993-2002, y en el tema del agua, 2003-2010, este trabajo intenta plasmar de manera preliminar las diferentes articulaciones observadas entre ambos fenómenos en la historia pero sobre todo en la actualidad. La contribución busca articular la reflexión a partir de las diferentes acepciones del concepto de frontera, de su interacción con el recurso hídrico y de los procesos migratorios observados en relación con las primeras y al segundo.

Para ello, hemos divido este trabajo en dos momentos. En primer lugar, a partir de dos acepciones del concepto de frontera, proponemos reflexionar en torno a la relación entre fronteras y agua. En segundo lugar, a partir de diferentes elementos empíricos encontrados a lo largo de diversas investigaciones en la frontera, exponemos las diferentes modalidades de la relación entre las migraciones y el agua en los escenarios fronterizos y transfronterizos de la región.

\section{Fronteras y agua entre México, Guatemala y Belice}

El concepto de frontera en español remite a varios términos en inglés que presentan significados distintos: la frontera como border, la frontera como frontier, y la frontera como boundary. Los dos primeros han sido conceptualizados por Jan de Vos, 1993 y 2005, a partir de la propia investigación de Turner (1893) y han sido utilizados para analizar los procesos históricos y contemporáneos de la frontera sur de México (De Vos, 1993, 2002a; Kauffer, 2005a, 2005b).
La frontera como border o frontera límite se refiere a la delimitación política entre soberanías nacionales, es decir, se trata de una frontera que divide y separa. Es la acepción política del concepto que se centra en la línea de división y en su delimitación, producto de acuerdos históricos o en algunos casos de conflictos. Generalmente, se caracteriza por su permanencia e inmovilidad a través de los años. ${ }^{2}$ Por su parte, la frontera como frontier remite a un proceso de colonización en el sentido de un frente que tiende a avanzar. Se relaciona con los sucesivos procesos de apropiación del territorio ${ }^{3}$ mediante los flujos de seres humanos, sus procesos de ocupación y los respectivos impactos de estos en los recursos naturales y, a diferencia de la anterior, se trata de una frontera que se transforma y se modifica constantemente. Finalmente, la frontera en el sentido de boundary se relaciona con las llamadas "fronteras secundarias" por Kearney (1999), mismas que han sido menos estudiadas en el contexto de la frontera entre México, Guatemala y Belice (Cruz, 1994; Kauffer, 2005a). Estas remiten a divisiones entre grupos culturales, lingüísticos, religiosos, sociales y étnicos, conceptualizadas por Barth (1969) como dicotomizaciones entre "nosotros", sobre la base de un origen común; y “ellos”, productos de la interacción social. Dicha conceptualización de la frontera no se desarrollará en este artículo. Así la primera parte de este trabajo evidenciará la presencia de agua en la frontera antes de analizar su importancia para las dos acepciones de frontera analizadas, la frontera límite y la frontera frente, y observar cómo las dimensiones de las fronteras límite y frente se encuentran a veces entrelazadas.

\section{El agua en la frontera entre México, Guatemala y Belice: una presencia multiforme}

Debido a las altas precipitaciones registradas en la región de la frontera entre México, Guatemala y Belice, que oscilan entre 1,237 $\mathrm{mm}$ anuales para el estado de 
Migraciones y AGUA EN LA FRONTERA ENTRE MÉXICO,

Quintana Roo y 2,095 mm en Tabasco (Conagua, 2010), pero que pueden llegar a 4,000 o 6,000 mm localmente en las zonas montañosas, por ejemplo en las partes altas de las cuencas en Guatemala (Dardón, 2002), ${ }^{4}$ la frontera se caracteriza por una presencia multiforme de recursos hídricos. En materia de aguas superficiales, la región mexicana administrativa hidrológica definida como frontera sur —estados de Chiapas, Tabasco, un municipio de Campeche y dos de Oaxaca - representa $36.8 \%$ de los escurrimientos superficiales de todo el país. Junto con la región administrativa conocida como península de Yucatán - que abarca todo el resto de Campeche, Quintana Roo y Yucatán-, ambas registran $53.6 \%$ de la recarga total de los acuíferos mexicanos y concentran $73.4 \%$ del agua renovable per cápita de todo México.

En la parte chiapaneca y tabasqueña de México y en gran parte de los departamentos guatemaltecos y distritos beliceños, encontramos numerosos recursos hídricos superficiales como ríos, lagos y humedales. Los dos ríos más caudalosos de México que concentran $46.4 \%$ del escurrimiento de la vertiente del Golfo y mar Caribe nacen en Guatemala y atraviesan el territorio de Chiapas y Tabasco antes de desembocar en el Golfo de México. En sus recorridos, se alimentan de numerosos afluentes existentes en los territorios de ambos países. Asimismo, se identifican seis cuencas transfronterizas que cubren gran parte del territorio de la región: las cuencas binacionales de los ríos Suchiate y Coatán, que nacen en Guatemala y desembocan en el Pacífico mexicano; la cuenca binacional del río Grijalva, que tiene su parteaguas en Guatemala y termina en el Golfo de México; la cuenca trinacional del río Usumacinta, que abarca 58\% de Guatemala, pero solamente $0.021 \%$ de Belice; la cuenca del río Candelaria, compartida por Guatemala y México y que desemboca en la Laguna de Términos en Campeche; la cuenca trinacional del río Hondo, que atraviesa el norte de Guatemala, antes de abarcar los territorios de Belice y México (ver mapa 1).
En estos escenarios fronterizos, los recursos hídricos presentan diferentes configuraciones geográficas con respecto a la ubicación de la línea de división internacional, algunos son fronterizos porque se ubican únicamente en algún lado de la frontera, otros son transfronterizos porque atraviesan la delimitación política, como varios humedales y numerosos ríos de diversos tamaños.

\section{La importancia del agua para la frontera límite}

Entre los numerosos ríos que se encuentran en la frontera entre México, Guatemala y Belice destacan los llamados ríos internacionales: el Suchiate, el Usumacinta y el Hondo, que tienen la particularidad de servir de marcadores para la delimitación de la frontera política. De hecho, su importancia es notable ya que 53\% de la frontera entre México y Guatemala son ríos y $87 \%$ de la frontera ente México y Belice corresponde al río Hondo. De tal forma que podemos afirmar que el agua es una dimensión fundamental de la frontera límite ya que sobresale en su modalidad de delimitación natural de frontera. Asimismo, en el caso de los tres ríos internacionales, su condición de frontera fluvial los hace muy distintos en términos de atención por parte de los Estados nacionales que todos los otros ríos que fluyen de un lado al otro de la línea de división internacional.

El río Suchiate marca la frontera en 77 de sus 92 kilómetros de recorrido. Por su parte, el río Usumacinta posee aproximadamente una tercera parte de sus 1,114 kilómetros que sirven de frontera entre México y Guatemala. Finalmente, de los 173 kilómetros del río Hondo, 160 delimitan la frontera entre México y Belice. ${ }^{5}$

La delimitación de ambas fronteras fue establecida a finales del siglo XIX por los tratados de límites firmados entre México y Guatemala en 1882, y México y Honduras Británicas en 1893. Sin embargo, en el caso de los ríos Suchiate y Hondo se trata de una delimitación problemática para la soberanía de los Estados debido a que los criterios que fueron propuestos 
para la delimitación de la frontera fluvial no permiten una adecuada fijación del límite internacional. Así, ambos tratados establecen que el thalweg, esto es, el canal más profundo del río, define la frontera entre México y Guatemala en los ríos Suchiate y Usumacinta, y entre México y Belice en el río Hondo. Se trata de un criterio utilizado en el siglo XIX para los ríos europeos cuando el uso de las aguas de los ríos internacionales era esencialmente la navegación y en el caso de los ríos caracterizados por poca fluctuación en su escurrimiento. Por lo tanto, es bastante adecuado para el río Usumacinta, el cual se encuentra en su mayor parte navegable y con un volumen estable. En el caso del río Suchiate, que nunca ha sido navegable y se caracteriza en la actualidad por un gasto que puede pasar de 10 en temporada de estiaje a 100 durante la estación de lluvias, no solamente la modalidad del thalweg resulta inadecuada en su búsqueda de equidad en materia de navegación sino provoca una considerable movilidad de la línea de división internacional ya que registra enormes fluctuaciones de volumen que se traducen en una movilidad del thalweg y, por ende, de la línea. De hecho, la situación problemática de esta frontera está en la orden del día desde 1940, fecha en la cual se iniciaron las primeras obras para contener el río, regresarlo a su cauce para evitar la movilidad de la frontera, y que sigue promovida por el gobierno mexicano hasta la actualidad.

Para el caso del río Hondo, que por ser una falla geográfica es en su mayor parte navegable, la situación inadecuada de la frontera definida por el thalweg se plantea en una zona de humedales de la parte alta de la cuenca donde el río tiene como nombre Arroyo Azul y corresponde a otro tipo de formación geológica. Como la denominación de arroyo lo indica, dicha corriente tiene un carácter intermitente y en temporada de estiaje el nivel del agua del Arroyo Azul disminuye tanto que dicha corriente no tiene cauce definido, en consecuencia su canal más profundo desaparece (CILA, 2005). Así, en esta parte, no es posible tener una frontera fluvial permanente ya que esta tiende a "evaporarse" en temporadas más secas. De hecho, hasta la fecha, no existe una completa demarcación física de las fronteras terrestre y fluvial entre México y Belice debido a que el tratado de límites de 1893 no especificó compromisos en materia de trazado, señalamiento y conservación de los límites (Auditoría Superior de la Federación, 2007). ${ }^{6}$

Si bien podemos afirmar que la presencia de los tres ríos internacionales es fundamental para la frontera límite, representa también en la actualidad, en los casos de los ríos Suchiate y Hondo, una problemática complicada para la línea política y para la soberanía de los Estados nacionales, ya que no asegura una permanencia y continuidad de la línea de división internacional.

\section{La presencia de agua como atracción: frentes de poblamiento y áreas despobladas}

En la acepción del concepto concebido como frontera frente, la región que México comparte con Guatemala y Belice evidencia múltiples facetas de una relación entre los recursos hídricos y las diferentes fases de poblamiento y de avance de la frontera agrícola en sus diversas partes. Así, en la historia y en la actualidad, la presencia de agua ha sidofundamental para las dinámicas relacionadas con la frontera frente. Adicionalmente, la ausencia de agua para el consumo humano ha sido un elemento clave para el despoblamiento del sur de los estados de Campeche y de Quintana Roo debido a la falta de recursos hídricos superficiales, como en el municipio campechano de Calakmul que colinda con Guatemala, y a las características químicas de las aguas de los ríos Candelaria y Hondo que no permiten su consumo (De Hernández, 2001; Herrera, 1946: 15, 17). En los tres escenarios fronterizos presentados, esta situación propició largos períodos de poco poblamiento del lado mexicano que terminaron entre los años sesenta a ochenta del siglo XX con procesos de colonización inducidos por el Estado mexicano. 
Migraciones y AGUA EN LA FRONTERA ENTRE MÉXICO,

Tomaremos tres ejemplos para ilustrar la relación entre la presencia de recursos hídricos y los flujos migratorios de avance de la frontera frente: la cuenca del río Usumacinta, y en particular el fenómeno de colonización de la selva Lacandona y del Petén guatemalteco; la cuenca del río Candelaria, y la cuenca del río Hondo. En estos tres casos, se aprecian procesos históricos y contemporáneos de frontera de colonización relacionados con la presencia de agua o incluso condicionados por su carencia.

La cuenca del río Usumacinta es la que presenta más elementos contundentes para analizar la relación entre los recursos hídricos y la frontera frente, sin duda porque se trata de una corriente sumamente caudalosa y también porque ha sido la cuenca más estudiada, principalmente del lado mexicano. Algunos arqueólogos reportan que las capitales regionales del mundo maya se ubicaban en lugares que tienen un fácil acceso al río Usumacinta, en particular debido a su papel en las comunicaciones dentro y fuera de la región (Aliphat, 1996: 27-28). En la época clásica, el segmento más sureño del río Usumacinta en México estaba articulado alrededor de la navegación con sus afluentes, mientras un segmento intermedio se organizaba en torno a asentamientos humanos cercanos a fuentes de agua que funcionaban como porteos entre rutas fluviales y terrestres y un tercero, ubicada más al norte formaba una serie de puertos de entrada a este sistema regional organizado en torno a la corriente principal, el Usumacinta, que fungía como un eje de interacción política, económica e histórica de la cultura maya clásica (Aliphat, 1996: 29).

La diferentes fase de la colonización de la selva Lacandona del lado mexicano han sido magistralmente expuestas por Jan de Vos en diversas publicaciones (De Vos, 1980, 1988a, 1988b, 1996, 2002b). A partir de éstas, retomamos los elementos de su obra que nos permiten hacer énfasis en la relación entre el agua, principalmente los ríos, el avance y el establecimiento de poblaciones en la zona. Si bien en un primer momento, la presencia de agua fue un obstáculo para la conquista española (De Vos, 1980: 48), en todos los períodos posteriores la frontera frente se desplazó gracias a las vías de comunicación fluviales y los principales centros de población se instalaron inicialmente en las orillas de los numerosos ríos. De hecho, los pobladores instalados en la zona en el período anterior al contacto, se ubicaban también en la proximidad de ríos y lagunas (De Vos, 1980). La historia moderna de la selva Lacandona inicia en 1822 con la apertura de las rutas fluviales a la navegación, las cuales sustentan la fase de explotación de la selva (De Vos, 1988a), en particular de la vegetación cercana a estos "caminos que andan" (De Vos, 1988a: 10). La importancia del conocimiento de los ríos y orillas por peteneros y tabasqueños fue fundamental para su avance en la selva (De Vos, 1988a: 42). Al igual que lo fue para los indios caribes, primeros habitantes de la selva, el Usumacinta siguió siendo el eje de la actividad económica de los períodos posteriores además de convertirse en el centro de su explotación por las monterías que lo utilizaban para transportar y exportar la madera hacia el Golfo de México, y se desplazaban río arriba para abastecer y establecer los asentamientos temporales. En la actual parte guatemalteca de la cuenca del Usumacinta, desde 1896 fueron otorgadas concesiones para la explotación de madera — caoba, cedro- y la instalación de monterías chicleras en la cuenca del río La Pasión, afluente del Usumacinta (Valdez, 2006). Al igual que en la selva Lacandona, la red hidrográfica fue determinante para la extracción de los recursos de la selva, y en particular Valdez (2006: 247) señala, además de los ríos La Pasión y Salinas otros cinco afluentes del Petén suficientemente caudalosos para permitir esta actividad de transporte hacia el río Usumacinta y el Golfo de México. Finalmente, al igual que en México, la instalación de monterías se realizaba en la proximidad de los ríos (Valdez, 2006 116). En la parte mexicana del Usumacinta, en los años cuarenta del siglo XX la apertura de nuevos frentes de 
colonización inició desde las cuencas de los ríos Jataté, Santo Domingo y las cañadas de sus afluentes (De Vos, 2002b: 31) - ver mapa 2-, cuando éstos se ampliaron en los años sesenta debido a movimientos espontáneos de colonización.

La relación entre poblamiento y agua en la cuenca del río Usumacinta - parte mexicana - vista por Jan de Vos (ver mapa 2).

Así, a mediados del siglo XX, la colonización de la selva por campesinos sin tierra y la explotación de los recursos naturales por compañías madereras privadas y luego por los gobiernos - centrados en las potencialidades de sus recursos hídricos - (De Vos, 2002b: 28) facilitaron el avance de la frontera frente. Del lado de Guatemala, De Vos (2002b: 52) subraya la promoción de cooperativas en las márgenes de los ríos Usumacinta, Chixoy y La Pasión para ocupar territorios despoblados ante las amenazas de proyectos de México y como estrategia de frontera frente para ocupar el espacio fronterizo de la frontera límite. Asimismo, De Vos (2002b: 52) evidencia la ubicación de las cooperativas del Ixcán a través de la promoción realizada por el gobierno y por grupos religiosos así como la formación de numerosos centros de población en las orillas de los ríos Chixoy, Tzejá, Xalbal e Ixcán entre los años 1966 y 1982 en el parteaguas más suroeste de esta misma cuenca en Guatemala próxima a la frontera. De la misma forma, presenta la edificación de cooperativas - mapa 3- en las orillas de los ríos La Pasión, Chixoy y Usumacinta.

Actualmente, el desarrollo de las comunicaciones terrestres ha desplazado el río Usumacinta en su papel de articulador de la economía regional. Sin embargo, la cercanía de las fuentes de agua sigue siendo un elemento fundamental para la supervivencia de la población en una región rezagada en acceso a los servicios públicos. Paralelamente, en la selva, el ecoturismo asociado al agua — ríos, lagunas, cascadas - y a los sitios arqueológicos, así como a los grupos indígenas, se ha desarrollado en fechas recientes principalmente en relación con los recursos hídricos.
La relación entre poblamiento y agua en la cuenca del río Usumacinta - parte guatemalteca- vista por Jan de Vos (ver mapa 3).

La cuenca del río Candelaria se ha caracterizado por un esquema similar al Usumacinta en cuanto al poblamiento: un auge prehispánico articulado al río como un eje de intercambios comerciales y de un sistema político económico en torno a Izamkanac, capital de Acalan, provincia chontal que articulaba un conjunto de 76 pueblos ubicados en las márgenes del río Candelaria y de su afluente el río Caribe (Vargas, 2001) desde finales del siglo XIV hasta 1571 cuando los españoles desplazan a toda la población hacia el norte. A partir de esta fecha, la zona se caracteriza por un período de despoblamiento seguido por una fase de extracción de recursos naturales centrada en el palo de tinte en los siglos XVII y XVIII y la explotación del chicle entre 1900 y 1940. El avance de la frontera frente culmina en un proceso de colonización articulado alrededor del río como medio de transporte y eje de instalación en los años sesenta del siglo XX. Los seis Nuevos Centros de Población Ejidal, NCPE, construidos por el gobierno mexicano entre 1963 y 1964 se realizaron en las orillas del río Candelaria (Revel-Mouroz, 1972: 239), situación que propició la comunicación fluvial entre estos asentamientos y durante los primeros años, antes de la construcción de la carretera. En todas las épocas, el río Candelaria fue un eje de comunicación y de desplazamiento de los flujos de población, en este sentido favoreció el avance de la frontera frente en torno a un centro político y económico durante las épocas prehispánicas, como eje de explotación de los recursos naturales a partir del siglo XVII y como área de instalación de colonizadores a partir de los años sesenta del siglo XX. Finalmente, al igual que en el río Usumacinta, hoy e el río Candelaria ya no es el eje de las comunicaciones regionales, tampoco de la vida política local, sino un paulatino elemento de promoción del ecoturismo a la par de una constante amenaza para las poblaciones que viven en sus orillas debido a las inundaciones. 
Migraciones y AGUA EN LA FRONTERA ENTRE MÉXICO,

Otro aspecto similar a la cuenca del río Usumacinta es la articulación entre el avance de la frontera frente y el reforzamiento de la frontera límite mediante la promoción de un proceso de colonización inducido por el Estado mexicano (Revel-Mouroz, 1972: 237) con la finalidad de reafirmar y proteger la parte mexicana de la línea de delimitación internacional. Del lado guatemalteco, no existen fuentes bibliográficas que permiten analizar el avance de la frontera frente en la historia ni en la actualidad, porque la zona se caracteriza por fenómenos incipientes de colonización en las márgenes del río en áreas de reservas naturales.

A finales del siglo XIX, la formación de la ciudad de Payo Obispo - hoy Chetumal, capital administrativa de Quintana Roo- fue una acción del Estado mexicano para afirmar su soberanía nacional en esta remota fracción del territorio (Chenaut, 1989: 13). En 1902 Quintana Roo adquirió la calidad de Territorio, a partir de esta fecha el Estado mexicano procuró ocupar la zona fronteriza para reforzar su presencia y su soberanía frente a la presencia inglesa, así como evitar el abastecimiento de armas a los mayas descendientes de la guerra de castas mediante un proceso de ocupación del territorio centrado en el control de las relaciones comerciales y en el otorgamiento de concesiones forestales (Macías, 2002).

El proceso de colonización de la cuenca del río Hondo promovido por el Estado se encaminó al poblamiento de la ribera del río alrededor de la actividad de explotación forestal y chiclera, que se organizó también en torno al río como eje de comunicación para la incursión a la selva y para el transporte de la madera. "Así, el río empezó a poblarse en sus orillas y al aglutinar a diversos centros de población" (Chenaut, 1989: 17) cuya existencia dependía del eje fluvial durante las primeras décadas del siglo XX. La dotación de ejidos entre los años 1920 y 1940 en la ribera mexicana del río Hondo permitió fijar centros de población, lo que facilitó el avance de la frontera frente (Dachary y Arnaiz, 1998). Uno de los objetivos del poblamiento del área consistió en hacer contrapeso al histórico dominio beliceño en la frontera. Finalmente, en los años setenta se inició una colonización dirigida por el Estado mexicano de las márgenes del río Hondo, con la intención de favorecer el desarrollo agrícola de la zona, ubicar a campesinos de otros estados demandantes de tierra y poblar, en aquel entonces, el territorio de Quintana para su conversión en estado, que se concretó en 1974. En este contexto se pueden distinguir tres momentos de avance de la frontera frente vinculados con tres flujos de migración que se articularon en torno a la presencia del río Hondo (Chenaut, 1989: 46), y que contribuyeron al poblamiento de su margen mexicana, del sur de Quintana Roo y de la frontera mexicana del río Hondo: las concesiones forestales, el reparto agrario de los años 1920-1940, y la colonización de los años sesenta.

Si bien hasta ahora disociamos los procesos de frontera límite y de frontera frente en su relación con el agua, los diferentes casos estudiados permiten también evidenciar cómo el avance de la frontera frente, favorecido por la presencia de agua para el consumo humano o para las comunicaciones locales y regionales, ha contribuido a un reforzamiento del límite de división internacional. Así, mediante el poblamiento de zonas poco habitadas y a través de distintos flujos de colonización, la demarcación internacional cobra cierta realidad y los Estados nacionales afirman su soberanía de tal forma que la frontera frente articulada con la presencia de recursos hídricos contribuye a la conformación de la frontera entre México, Guatemala y Belice, principalmente en las márgenes mexicanas y guatemaltecas del Usumacinta y sus afluentes, en la ribera mexicana del Hondo y en la porción mexicana del Candelaria. En este proceso, el papel de los Estados y sus esfuerzos de colonización dirigida fueron clave, aunque algunos otros actores también participaron en este doble fenómeno, como las compañías madereras en el deslinde de los límites entre 1882 y 1895 en los ríos San Pedro Mártir, Usumacinta, Lacantún, Chixoy, La Pasión, Tzendales y el Alto Usumacinta, todos ubicados 
en el territorio conocido como "cuenca del Usumacinta" (De Vos, 1988a).

Agua y migraciones: una relación ambivalente en la frontera

Más allá de la relación entre los recursos hídricos y los flujos migratorios bajo la modalidad de olas de colonización en el sentido de una frontera de apropiación del territorio y de conformación de un límite político, las interacciones entre el fenómeno migratorio y el agua en la frontera entre México, Guatemala y Belice, son múltiples. Para entender esta diversidad, es necesario describir brevemente las migraciones en la región antes de analizar cómo el agua y las personas convergen, cómo su coincidencia facilita los cruces, y cómo su exceso favorece los desplazamientos humanos.

\section{La frontera entre México, Guatemala y Belice: un mosaico de migraciones}

Las distintas migraciones contemporáneas en la frontera entre México, Guatemala y Belice, transitan, se originan y tienen como destino este territorio de tal forma que tienden a entrelazarse. Representan a la vez el resultado de acontecimientos históricos como la propia delimitación de la frontera política a finales del siglo XIX y de procesos contemporáneos económicos, agrarios, políticos, así como más recientemente también el resultado de eventos ambientales brutales o de deterioros acumulados. Se trata de un fenómeno social, político y económico, cada día más estudiado en sus diferentes modalidades de flujos internos, regionales, internacionales y transnacionales, el cual, después de muchos años de estudios centrados únicamente en el estado de Chiapas, empieza a nutrirse de trabajos recientes realizados en Tabasco (Arriola, 2009; Vautravers y Ochoa, 2009), Campeche y Quintana Roo.

En la frontera de estudio podemos distinguir las migraciones económicas — que obedecen a la búsqueda de mejores oportunidades de trabajo o de condiciones de vida- de las políticas - motivadas por factores políticos hoy poco representativas después del término oficial del refugio guatemalteco en México en 1999, que cerró un proceso de casi dos décadas-, y de las migraciones favorecidas por las condiciones ambientales entendidas como desastres súbitos o deterioros acumulados. La delimitación entre estos tres "tipos ideales" no es siempre sencilla, ya que los factores suelen estar entrelazados. Sin embargo, permiten identificar distintos factores asociados a los flujos migratorios. Estas tres grandes categorías de migraciones se combinan con otro tipo de características con respecto a la frontera -migraciones internas o internacionales-, a los espacios locales expulsores, receptores o de paso y sus combinacionesy al momento del proceso - migración a Estados Unidos, migración de retorno, expulsión- además de su carácter individual, colectivo, con o sin documentos. Así podemos observar situaciones de emigración en muchas áreas de Guatemala y más recientemente en Chiapas y Tabasco, flujos de inmigración en particular en el estado de Quintana Roo y en menor medida en el estado de Campeche, fenómenos de transito en el norte de Guatemala y tradicionalmente en los estados de Chiapas, más recientemente en Tabasco y Campeche con el desplazamiento de las rutas que atravesaban el estado de Chiapas hacia el oriente (Kauffer, 2009).

\section{Las aguas que fluyen y flujos humanos: convergencias}

En estos escenarios fronterizos, la frontera límite hoy está lejos todavía de representar una barrera debido a la existencia de un contínuum cultural histórico, de relaciones familiares, comerciales y de intercambios cotidianos así como de cruces constantes de un lado al otro, situación que nos permite afirmar que más que un límite real se trata de una línea imaginaria en los hechos. Esta porosidad de la frontera límite 
favorece los flujos migratorios transfronterizos. Así, al igual que los recursos hídricos fluyen sin respetar las fronteras establecidas por los seres humanos, los flujos migratorios se caracterizan por transitar vía estos recursos naturales que escurren de un país a otro. En consecuencia, el agua se convierte en el soporte de varios de los flujos migratorios internacionales y favorece el tránsito de personas a través de la frontera límite y en los espacios fronterizos. Del Pacífico al Caribe, encontramos en primer lugar el río internacional Suchiate en las márgenes del cual se desarrolla una de las economías transfronterizas más activas. El Suchiate se encuentra en la ruta migratoria más antigua de la frontera en donde tradicionalmente los migrantes abordan el tren con el cual atraviesan todo México. La principal modalidad de cruce son las balsas, rústicas embarcaciones elaboradas con llantas y madera, pero es posible en temporada de estiaje atravesar el río caminando o en bicicleta. Por su parte, el río Usumacinta en su porción chiapaneca y tabasqueña ha adquirido recientemente importancia como consecuencia del paulatino desplazamiento de los flujos migratorios hacia el oriente de la frontera y facilita algunos cruces que por su fuerte caudal requieren la colaboración de los dueños de las lanchas que son alquiladas de forma individual o grupal. Asimismo, el río Candelaria ha inaugurado una ruta migratoria internacional conocida como la ruta del río (Zapata Bosch, s/f), la cual obedece a la lógica de desplazamiento de los flujos migratorios antes mencionado hacia el este aunque todavía no registra grandes flujos. Finalmente, en el río Hondo, que representa la segunda zona de actividad económica más activa de toda la frontera, si bien la migración internacional es escasa existe un constante tránsito local de personas y mercancías de un lado al otro de la frontera límite.

Cabe subrayar que si bien la coincidencia entre migraciones internacionales y recursos hídricos se plantea de manera favorable en la frontera entre México, Guatemala y Belice por su carácter facilitador de los flujos, no está exenta de riesgos para los migrantes ya que en ocasiones las embarcaciones colapsan o las personas son arrastradas por las corrientes de los ríos. En los dos ríos más utilizados por los migrantes, el Suchiate y el Usumacinta, se han registrado accidentes y muertes de personas que recurren a la vía fluvial en su paso por México. La relación entre recursos hídricos y flujos migratorios en tanto los primeros facilitan los segundos se establece vía la frontera límite, su característica de porosidad y su componente fluvial. Asimismo, se realiza a través de dicha frontera mediante el uso de ríos y cuerpos de agua que atraviesan el límite político, y se establece en el área fronteriza debido a su abundancia.

\section{Los desplazamientos inducidos por los excesos de agua: desastres y migraciones}

La frontera entre México, Guatemala y Belice, rica en recursos hídricos como resultado de sus elevadas precipitaciones es también un escenario caracterizado por los llamados fenómenos hidrometeorológicos extremos bajo la forma de huracanes, tormentas tropicales, que provocan lluvias torrenciales concentradas en pocos días, bruscos aumentos de los volúmenes de los ríos e inundaciones en las planicies, zonas costeras e incluso en áreas urbanas ubicadas de manera inadecuada con respecto a los ríos. Dicha situación se produce en diferentes puntos de las seis cuencas que México comparte con Guatemala y Belice. A este respecto, podemos mencionar los desastres de 1998 y 2005 en la costa de Guatemala y Chiapas que resultaron del huracán Mitch y las lluvias asociadas al Stan, las inundaciones de 1999 y 2007 en la parte tabasqueña de la cuenca del río Grijalva y las ocurridas en 2008 en las cuencas de los ríos Usumacinta y Candelaria. A la par de estos fenómenos que impactan a gran escala, encontramos situaciones locales de colonias o áreas urbanas limitadas donde las inundaciones son bastante recurrentes. En las áreas montañosas, las altas precipitaciones producen aludes, deslaves y están 
asociadas al deterioro de la cobertura vegetal de estos territorios (Dardón y Morales, 2006). Así, mientras las cuencas de los ríos Suchiate y Coatán presentan solamente $23.73 \%$ y $23.87 \%$ de cobertura de bosques y selvas con únicamente $0.25 \%$ en el departamento de Huehuetenango en Guatemala para esta última cuenca; la del río Grijalva registra $42.83 \%$ con un deterioro muy marcado en Tabasco, 5.79\%. ${ }^{7}$ Por su parte la cuenca del río Grijalva evidencia una presencia de bosques y selvas equivalente a $45.24 \%$ de su territorio con una tendencia muy baja en Tabasco, 9.72\%. Las más conservadas son las cuencas de los ríos Candelaria y Hondo con $61.66 \%$ y $66 \%$ de vegetación, pero en la última la zona que corresponde al distrito de Corozal en Belice solamente reporta 4.87\% de cobertura de bosques y selvas. En este contexto que combina altas precipitaciones y procesos de deterioro de algunas partes altas de las cuencas -Coatán y Suchiate- o de áreas de planicie - Tabasco-, la abundancia de precipitaciones favorece la expulsión de las poblaciones que viven en estos territorios porque no permiten la continuidad de las actividades agrícolas debido al deterioro de los suelos o como consecuencia de la erosión. En otros casos, las inundaciones amenazan la vida de los habitantes de los márgenes de los ríos e interrumpen sus actividades económicas, agrícolas o comerciales, y producen considerables daños en las infraestructuras de los servicios públicos. También acentúan la aguda vulnerabilidad de la población migrante al ocurrir durante el propio proceso migratorio (Centro de Derechos Humanos Fray Matías de Córdova, A. C., 2006).

En este caso, la relación entre agua, migración y frontera, remite principalmente a la frontera como escenario de colonización y a las consecuencias que dicho proceso característico de las últimas décadas ha tenido en la erosión de los suelos, en la deforestación y en consecuencia la limitada capacidad actual de muchos ecosistemas de las cuencas transfronterizas para retener el agua, como por ejemplo, en el río Suchiate.
De esta manera, las interacciones entre los recursos hídricos y los flujos migratorios en los escenarios fronterizos ubicados de ambos lados de la frontera entre México, Guatemala y Belice, resultan múltiples: a veces el agua es un vector de los desplazamientos, en otros casos favorece la expulsión de las poblaciones o incluso puede representar un riesgo.

\section{Conclusión}

La frontera entre México, Guatemala y Belice, mejor conocida bajo la denominación hegemónica de "frontera sur" por la hoy numerosa producción académica mexicana, aunque incompleta porque no cubre ni todos los fenómenos contemporáneos ni la totalidad de su extensión, evidencia la existencia de fenómenos concurrentes en materia de migración y de agua. Castillo et al. (2006: 8) mencionan que "hacer una historia de la frontera sur de México requiere un esfuerzo de visión transfronteriza de los hechos y procesos históricos que han tenido lugar en ambas márgenes de los ríos Hondo, Suchiate y Usumacinta". Este trabajo intenta seguir la sugerencia temática anterior, por lo tanto parte de un reconocimiento de la importancia de los ríos internacionales para la frontera límite y propone ampliar la mirada al papel de los recursos hídricos en las olas de poblamiento de la frontera frente. De igual forma, la contribución trata de abordar las problemáticas planteadas desde una visión transfronteriza, aunque debemos reconocer que la poca disponibilidad de antecedentes bibliográficos para las áreas beliceña y guatemalteca de la frontera dificulta el desarrollo de un enfoque realmente incluyente.

Finalmente, a partir de una primera reflexión en torno al agua y las fronteras, el trabajo se centra en la exploración preliminar de interacciones entre el recurso hídrico y las migraciones en los escenarios fronterizos de la región y en sus relaciones con ambos tipos de frontera. En este caso, únicamente esboza algunas confluencias y relaciones que merecerían un trabajo 
más detallado y profundo. Ello evidencia que la frontera entre México, Guatemala y Belice, posee en la actualidad amplios campos de investigación que explorar en sus diferentes dimensiones ambientales y sus respectivas interacciones con fenómenos político sociales.

\section{Notas}

${ }^{1}$ Cabe subrayar que la visión de la frontera sur, mexicana y excluyente con respecto a los vecinos del sur, predomina entre las instancias gubernamentales mexicanas como lo indican el Programa-Hídrico Ambiental de la Frontera Sur y la Comisión Binacional del río Hondo promovidos en el año 2009 por la Comisión Nacional del Agua, así como el Programa Integral para la Frontera Sur, impulsado por el gobierno federal mexicano en 2010. En estos tres casos, la dimensión fronteriza se centra en los intereses mexicanos y carece por lo tanto de visión transfronteriza y regional.

${ }^{2}$ Como lo describiremos más adelante, esta inmovilidad de la frontera límite es relativa en el caso de las fronteras fluviales de los ríos Suchiate y Hondo.

${ }^{3} \mathrm{Si}$ bien existe una relación entre el concepto de frontera y de territorio como construcciones sociales, consideramos que la discusión en torno al territorio en su relación con los recursos hídricos y sus interacciones con los flujos migratorios requiere una aproximación más local en tanto se entiende como construido y apropiado por los diferentes actores. La perspectiva eminentemente macro del presente trabajo hace difícil la incorporación de la noción de territorio para el análisis en un espacio tan amplio geográficamente y tan diverso culturalmente hablando que es atravesado por flujos migratorios de distinta índole.

${ }^{4}$ Los datos más actualizados y confiables que tomamos como referencia son mexicanos, pero de igual forma las partes guatemalteca y beliceña registran altas precipitaciones y una gran presencia de agua. Cabe subrayar que los estados de Tabasco, Chiapas, Campeche y Quintana Roo, ocupan respectivamente los lugares 1, 2, 4 y 5 en precipitación a escala nacional en México (Conagua, 2010) frente a un promedio de $760 \mathrm{~mm}$. No referimos a información guatemalteca y beliceña debido a su inexistencia.

${ }^{5}$ Cabe subrayar que estas cifras fueron calculadas a partir de mapas en un sistema de información geográfica, SIG pero según los datos que reportan los tratados que han sido retomados recientemente por la Auditoría Superior de la Federación, en 2007, la frontera fluvial entre México y Belice representaría 94.7\%, 252 de 266 $\mathrm{km}$, y estaría formada por $80 \mathrm{~km}$ de frontera marítima - no acordada entre ambos países - en la Bahía de Chetumal, 119 km del río Hondo, y $53 \mathrm{~km}$ del Arroyo Azul. En cuanto a la frontera entre México y Guatemala, la Auditoría Superior de la Federación, también en 2007, menciona que solamente $40.3 \%$ es fluvial, es decir $386.6 \mathrm{~km}$ de ríos de los cuales 82 corresponden al río Suchiate y lo demás - sin mencionar con precisión la longitud - corresponden a los ríos Usumacinta y Salinas. Asimismo, a diferencia de otras fuentes académicas publicadas que hablan de 1,139 km de frontera entre México y sus vecinos del sur (Castillo et al., 2006) o 1,149 km (Hernández Daumás, 2005; Conapo, 2010), la Auditoría Superior de la Federación, datos de 2007, revela que su longitud es de 1,225.6 km. Como lo menciona Jan de Vos (1994: 10), los mapas con los cuales fueron establecidos los tratados de límites a finales del siglo XIX resultaron muy aproximados, de tal forma que no parecen una base confiable frente a los SIG actuales.

${ }^{6}$ Es fundamental mencionar que dicho tratado de 1893 no considera a Belice como Estado independiente ya que era en aquel entonces colonia de Honduras Británica, es decir, que el tratado fue firmado entre México, Gran Bretaña e Irlanda, y que un tratado nuevo ha sido sugerido sin registrar avances (Auditoría Superior de la Federación, 2007). Adicionalmente a la ausencia de delimitación física fija de la línea terrestre y a la problemática de evaporación de la frontera fluvial en el Arroyo Azul, el trazo de la línea divisoria en la bahía 
de Chetumal propuesto por México en el año 2006 no ha sido validado por Belice (Auditoría Superior de la Federación, 2007).

${ }^{7}$ Los datos presentados proceden de un sistema de información geográfica derivado de la delimitación de las seis cuencas transfronterizas entre México, Guatemala y Belice a partir de la información del Instituto Nacional de Información Geográfica y Estadística (Inegi) de México y del Instituto Nacional de Sismología, Vulcanología, Meteorología e Hidrología (Insivumeh) de Guatemala.

\section{Bibliografía}

Aliphat Fernández, Mario M. (1996), “Arqueología y paisajes del Alto Usumacinta”, en Arqueología Mexicana, vol. IV, núm 22, pp-24-29.

Ángeles, Hugo, Laura Huicochea, Antonio Saldívar y Esperanza Tuñón (coordinadores) (2005), Actores y realidades en la frontera sur de México, México: El Colegio de la Frontera Sur, Consejo Estatal de Población.

Arriola Vega, Luis Alfredo (2009), "Seguridad y migración en el espacio fronterizo Tabasco-El Petén", en Migración y desarrollo, segundo semestre, pp. 27-45.

Auditoría Superior de la Federación (2007), Auditoría de Desempeño sobre el Cumplimiento de los Tratados de Límites con Guatemala y Belice, Cuenta Pública 2007, México: Auditoría Superior de la Federación.

Barth, Fredrik (1969), Ethnics Groups and Boundaries. The Social Organization of Culture Difference, Bergen: Oslo, Universitetsforlaget.

Basail Rodríguez, Alain (coordinador) (2005), Fronteras des-bordadas. Ensayo sobre la frontera sur de México, México: Casa Juan Pablos, Universidad de Ciencias y Artes de Chiapas.

Castillo, Manuel Ángel, Mónica Toussaint Ribot y Mario Vázquez Olivera (2006), Espacios diversos, historia en común, México, Guatemala y Belice, La construcción de una frontera, México: Secretaría de Relaciones Exteriores. Centro de Derechos Humanos Fray Matías de Córdova
A.C. (2006), Huracân Stan: "la dignidad devastada", Informe alternativo sobre afectaciones y violaciones a derechos humanos de las personas afectadas por el Huracán Stan, en comunidades de la Costa, Sierra y Frontera del Estado de Chiapas, Tapachula: Centro de Derechos Humanos Fray Matías de Córdova A. C.

Chenaut, Victoria (1989), Migrantes y aventureros en la frontera sur, México: Secretaría de Educación Pública, Centro de Investigaciones y Estudios Superiores en Antropología Social.

Comisión Internacional de Límites y Aguas (CILA) (2005), Problemas de la delimitación fluvial de México en Guatemala y Belice, Ponencia presentada en el Segundo Congreso Internacional sobre Agua en la Frontera México-Guatemala-Belice, Campeche, Campeche, 7 y 8 de diciembre.

Comisión Nacional del Agua (CONAGUA), Estadísticas del agua 2010, México: CONAGUA.

Cruz Burguete, Jorge Luis (1998), Identidades en fronteras, fronteras de identidades. Elogio de la intensidad de los tiempos en los pueblos de la frontera sur, México: El Colegio de México.

Dachary, Alfredo César y Stella Maris Arnaiz Burne (1998), El Caribe mexicano. Una frontera olvidada, México: Universidad de Quintana Roo, Fundación de Parques y Museos de Cozumel.

Dardón S., J. Jacobo (coordinador) (2002), Caracterización de la frontera de Guatemala/México, Guatemala: Editorial de Ciencias Sociales, Facultad Latinoamericana de Ciencias Sociales.

Dardón, Jacobo y Cecilia Morales (coordinadores) (2006), ¿Por qué tanta destrucción? Las amenazas naturales y estructurales: sistematización de la vulnerabilidad, le negligencia y la exclusión regional del altiplano occidental den la tormenta asociada Stan, Guatemala: Editorial de Ciencias Sociales, Movimiento Tzuk Kim-Pop.

De Vos, Jan (1980), La paz de Dios y del Rey. La conquista de la Selva Lacandona (1525-1821), México: Secretaría de Educación y Cultura de Chiapas, Fondo de Cultura Económica. 
Migraciones y AGUA EN LA FRONTERA ENTRE MÉXICO,

De Vos, Jan (1988a), Oro verde. La conquista de la Selva Lacandona por los madereros tabasqueños, 1822-1949, México: Gobierno del estado de Tabasco, Fondo de Cultura Económica.

De Vos, Jan (1988b), Viajes al Desierto de la Soledad. Cuando la Selva Lacandona aún era selva, México: Secretaría de Educación Pública, Centro de Investigaciones y Estudios Superiores en Antropología Social.

De Vos, Jan (1993), Las fronteras de la frontera sur. Reseña de los proyectos de expansión que figuraron la frontera entre México y Centroamérica, México: Universidad Juárez Autónoma de Tabasco, Centro de Investigaciones y Estudios Superiores en Antropología Social.

De Vos, Jan (1996), "Donde abundaron los monos sagrados. Semblanza del río Usumacinta”, en Arqueología Mexicana, vol. IV, núm. 22, pp-54-63.

De Vos, Jan (2002a), "Las fronteras de la frontera sur. Una visión histórica”, en Edith F. Kauffer Michel (editora) (2002), Identidades, migraciones y género en la frontera sur de México, México: El Colegio de la Frontera Sur, pp. 49-67.

De Vos, Jan (2002b), Una tierra para sembrar sueños. Historia reciente de la Selva Lacandona, 1950-2000, México: Centro de Investigaciones y Estudios Superiores en Antropología Social, Fondo de Cultura Económica.

Fábregas Puig, Andrés (2005), "Elconceptodefrontera: una formulación”, en Alain Basail Rodríguez (coordinador), Fronteras des-bordadas. Ensayo sobre la frontera sur de México, México: Casa Juan Pablos, Universidad de Ciencias y Artes de Chiapas, pp. 21-5l.

Hernández Daumás, Salvador (Coord.) (2005), Frontera sur de México. Cinco formas de interacción entre sociedad y ambiente, México: El Colegio de la Frontera Sur.

Hernández, Margarita P. (2001), Cerca de un manantial, El agua y el desarrollo de Quintana Roo, Chetumal: Instituto Quintanarroense de la Cultura.

Herrera, Horacio (1946), Monografía del río Hondo, Quintana Roo, E.U. Mexicanos, México: Sociedad Mexicana de Geografía y Estadísticas.
Kauffer Michel, Edith F. (2002a), "Movimientos forzosos en la frontera sur. Una visión comparativa de los refugiados guatemaltecos en el sureste mexicano", en Edith F. Kauffer Michel (Ed.), Identidades, migracionesy género en la frontera sur de México, México: El Colegio de la Frontera Sur, pp. 215-242.

Kauffer Michel, Edith F. (2005a), "De la frontera política a las fronteras étnicas: refugiados guatemaltecos en México", en Frontera Norte, núm. 34, pp. 7-36.

Kauffer Michel, Edith F. (2009), "Mujeres indígenas, procesos migratorios y derechos humanos en la frontera sur de México", en Instituto Interamericano de Derechos Humanos, Las mujeres indígenas de América Latina y los procesos migratorios, San José, Costa Rica: Instituto Interamericano de Derechos Humanos, pp. 87-105.

Kauffer Michel, Edith F. (editora) (2005b), El agua en la frontera México-Guatemala, Belice, México: El Colegio de la Frontera Sur, UNACH, RISAF, TNC, The David and Lucile Packard Foundation.

Kauffer Michel, Edith F. (editora) (2002b), Identidades, migraciones y género en la frontera sur de México, México: El Colegio de la Frontera Sur, p. 270.

Kearney, Michael (1999), "Fronteras fragmentadas, fronteras reforzadas", en Gail Mummert (editora), Fronteras fragmentadas, Morelia: El Colegio de Michoacán, CIDEM, pp. 559-570.

Limón Aguirre, Fernando (2008), "La ciudadanía del pueblo chuj en México. Una dialéctica negativa de identidades", en Alteridades, vol. 18, núm. 35, pp. 85-98.

Macías Zapara, Gabriel Aaarón (2002), La península fracturada. Conformación marítima, social y forestal del Territorio Federal de Quintana Roo. 1884-1902, México: Centro de Investigaciones y Estudios Superiores en Antropología Social, Miguel Ángel Porrúa.

Morales Bermúdez, Jesús (2005), "La frontera sur en su literatura. Notas para su estudio", en Alain Basail Rodríguez (coordinador), Fronteras des-bordadas. Ensayo sobre la frontera sur de México, México: Casa Juan Pablos, Universidad de Ciencias y Artes de Chiapas, pp. 53-85. 
Reval-Mouroz, Jean (1972), Aprovechamiento y colonización del trópico húmedo mexicano, México: Fondo de Cultura Económica.

Valdez Gordillo, Mario E. (2006), Desencuentro y encuentro de fronteras. El Petén guatemalteco y el Sureste mexicano, Tuxtla Gutiérrez: Universidad de Ciencias y Artes de Chiapas, Universidad Intercultural de Chiapas.

Vargas, Ernesto (2001), Itzamkanacy Acalan. Tiempos de crisis anticipando el futuro, México: Universidad Nacional Autónoma de México.

Vautravers Tosca, Guadalupe (2005), Estudio comparativo de la frontera Tabasco, México-El Petén, Guatemala,
Villahermosa: Universidad Juárez Autónoma de Tabasco.

Vautravers Tosca, Guadalupe y Aurora Marianela Ochoa Rivera (2009), "Las recientes transformaciones de la migración en Tabasco", en Migraciones Internacionales, vol. 5, núm. 1, pp. 65-91.

Villafuerte Solís, Daniel (2004), La frontera sur de México. Del TLC México-centroameérica al Plan Puebla-Panamá, México: Universidad Nacional Autónoma de México, Plaza y Valdés.

Zapata Bosch, (s/f), la ruta del río 〈http://www. campechedigital.com/writer.php?n=5\&a=951> [20 de enero 2010].

Mapa 1. La frontera entre México, Guatemala y Belice, y las cuencas compartidas

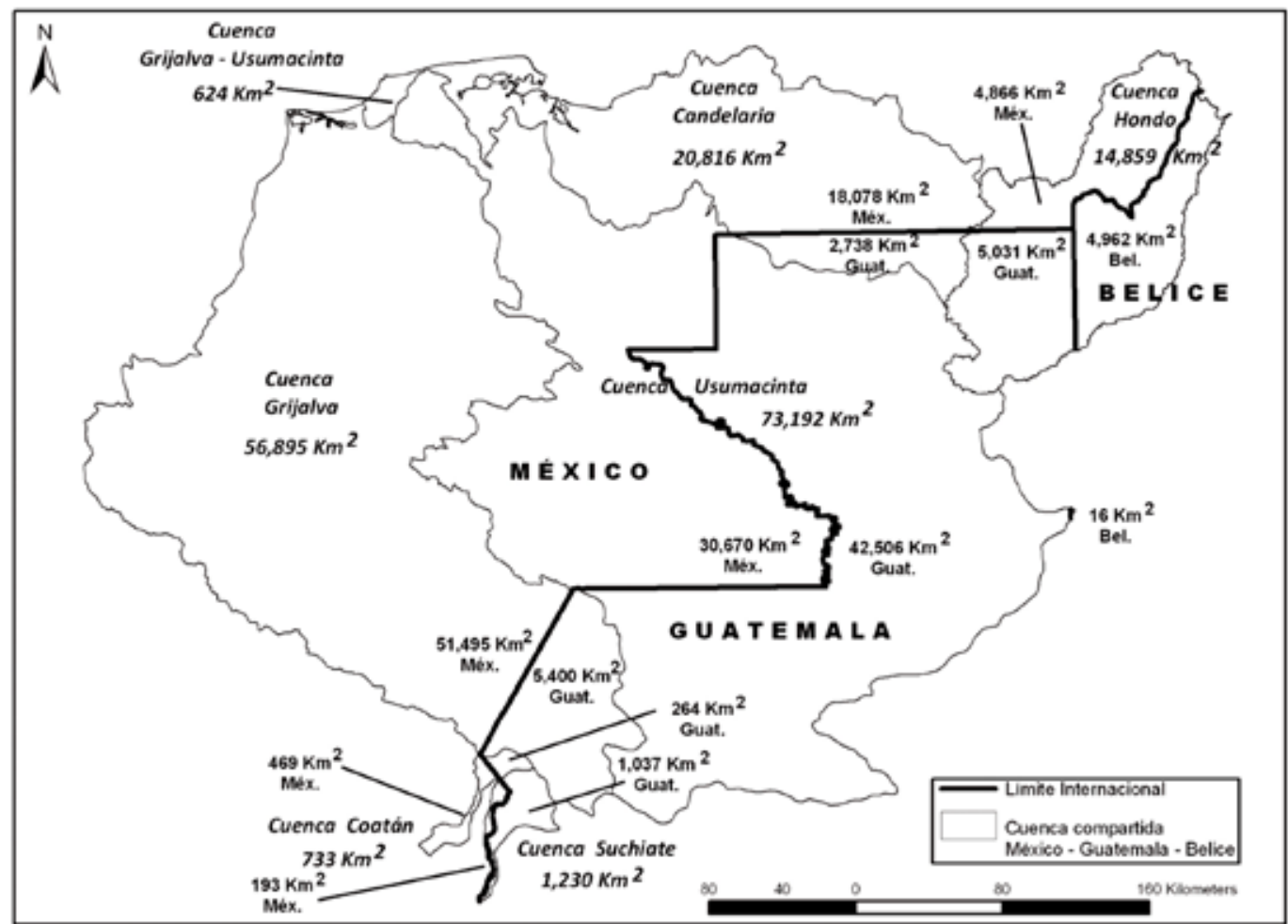

Fuente: elaborado por Antonino García y Emmanuel Valencia (2010). 
Mapa 2. Poblamiento de la Cuenca del Río Santo Domingo, 1960-1970.

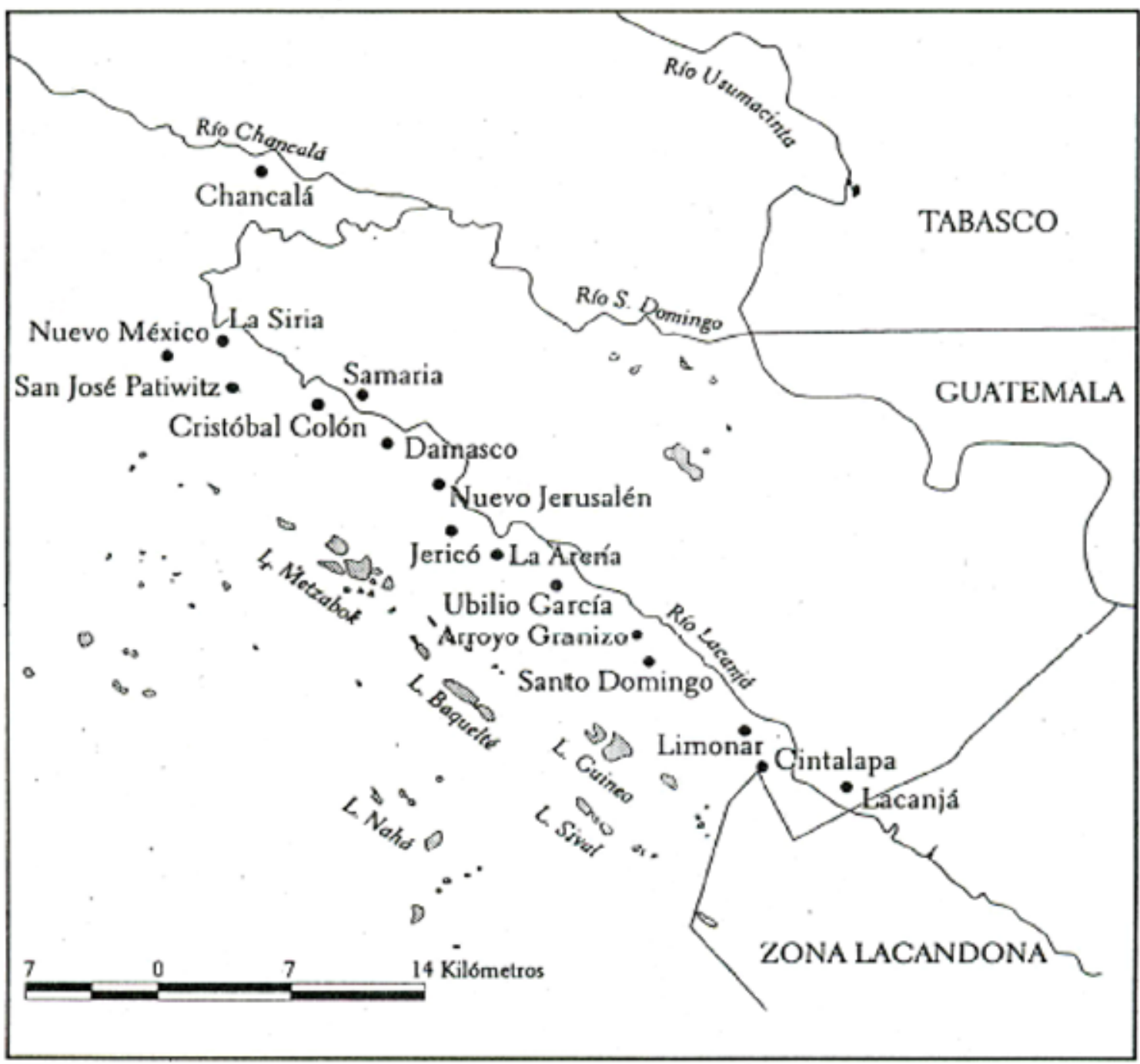

Fuente: De Vos (2002b). 
Mapa 3. Cooperativas campesinas de los ríos La Pasión y Usumacinta, en El Petén.

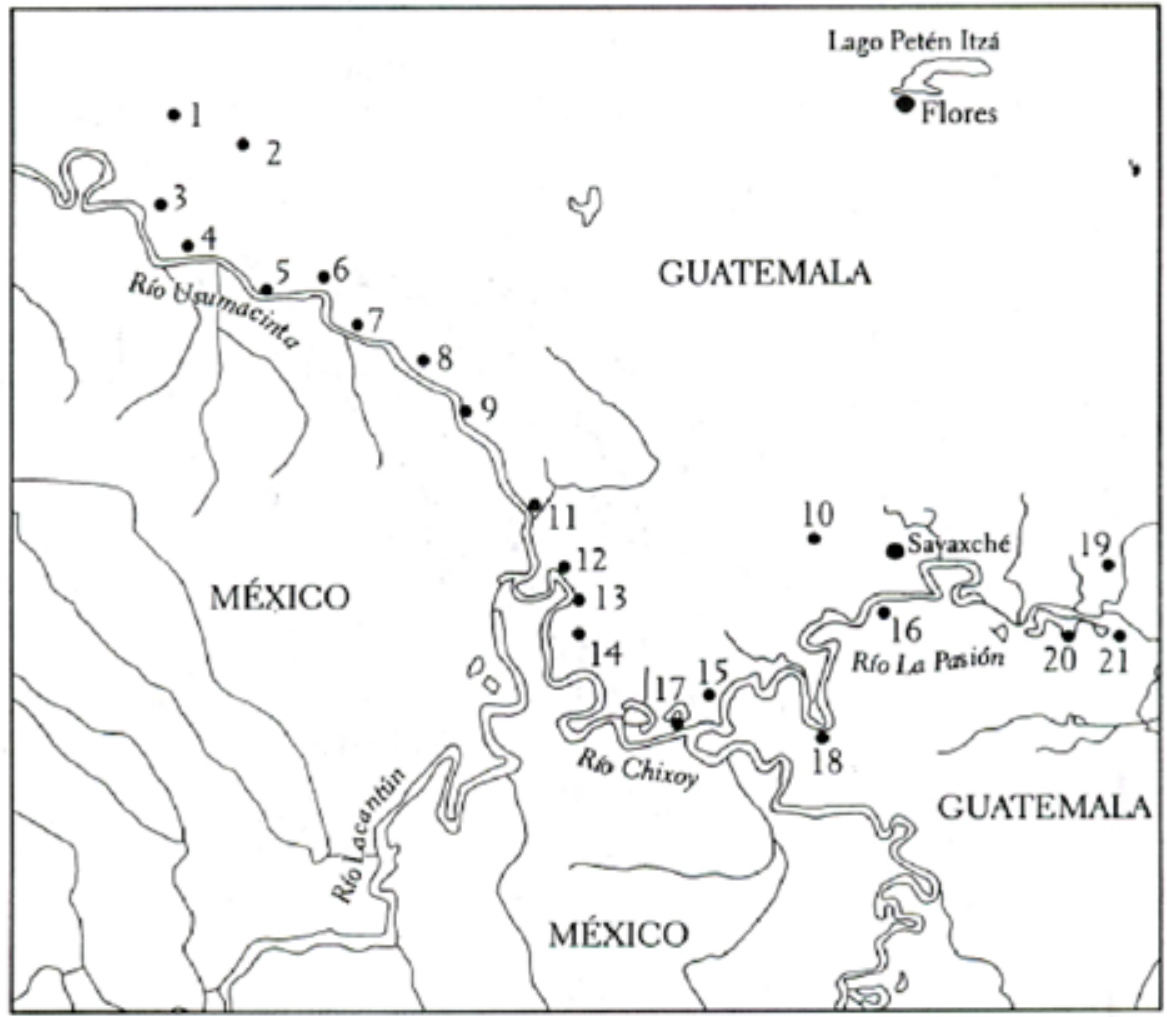
1. Quetzal
2. La Lucha
3. Centro Campesino
4. Técnica Agropecuaria
5. La Nueva Felicidad
6. Bethel
7. Monte Sinaí

8. Bonanza

9. El Arbolito

10. La Palma

11. La Bella Guatemala

12. Flor de la Esperanza

13. Ixmucané

14. Los Ángeles
15. Buena Fe

16. Manos Unidas

17. El Sacrificio

18. Mario Méndez

19. La Amistad

20. Las Flores

21. El Caserío

Fuente: De Vos (2002b). 